\title{
Far and proximity maneuvers of a constellation of service satellites and autonomous pose estimation of customer satellite using machine vision
}

\author{
Gilberto Arantes Jr. ${ }^{a}$, Evandro Marconi Rocco ${ }^{\mathrm{b}, *}$, Ijar M. da Fonseca ${ }^{\mathrm{b}}$, Stephan Theil ${ }^{\mathrm{c}}$ \\ a Center of Applied Space Technology and Microgravity - ZARM, Germany \\ ${ }^{\mathrm{b}}$ National Institute for Space Research - INPE, Avenida dos Astronautas, 1758, Space Mechanics and Control Division, CEP 12227-010 São José dos Campos, \\ SP, Brazil \\ ${ }^{\mathrm{c}}$ Germany's National Research Center for Aeronautics and Space - DLR, Germany
}

\section{A R T I C L E I N F O}

\section{Article history:}

Received 6 February 2009

Received in revised form

28 October 2009

Accepted 29 November 2009

Available online 13 January 2010

\section{Keywords:}

Rendezvous and docking

On-orbit servicing

Orbital maneuver

Satellite constellation

Computer vision

Pose estimation

\begin{abstract}
A B S T R A C T
Space robotics has a substantial interest in achieving on-orbit satellite servicing operations autonomously, e.g. rendezvous and docking/berthing (RVD) with customer and malfunctioning satellites. An on-orbit servicing vehicle requires the ability to estimate the position and attitude in situations whenever the targets are uncooperative. Such situation comes up when the target is damaged. In this context, this work presents a robust autonomous pose system applied to RVD missions. Our approach is based on computer vision, using a single camera and some previous knowledge of the target, i.e. the customer spacecraft. A rendezvous analysis mission tool for autonomous service satellite has been developed and presented, for far maneuvers, e.g. distance above $1 \mathrm{~km}$ from the target, and close maneuvers. The far operations consist of orbit transfer using the Lambert formulation. The close operations include the inspection phase (during which the pose estimation is computed) and the final approach phase. Our approach is based on the Lambert problem for far maneuvers and the Hill equations are used to simulate and analyze the approaching and final trajectory between target and chase during the last phase of the rendezvous operation. A method for optimally estimating the relative orientation and position between camera system and target is presented in detail. The target is modelled as an assembly of points. The pose of the target is represented by dual quaternion in order to develop a simple quadratic error function in such a way that the pose estimation task becomes a least square minimization problem. The problem of pose is solved and some methods of non-linear square optimization (Newton, Newton-Gauss, and Levenberg-Marquard) are compared and discussed in terms of accuracy and computational cost.
\end{abstract}

(c) 2009 Elsevier Ltd. All rights reserved.

\section{Introduction}

Spacecraft rendezvous and docking/berthing (RVD) autonomous operations are very important in the context of on orbit servicing (OOS) satellites. A servicing satellite

\footnotetext{
* Corresponding author. Tel.: +55123945 6192 .

E-mail address: evandro@dem.inpe.br (E.M. Rocco).
}

requires the ability to rendezvous and dock without preliminary information of the customer vehicle, because in such a case the vehicle could be damaged or out of service, such satellite is referred to as non-cooperative. They can be categorized in future assets, satellites designed to be service (e.g. with orbital replacement units, grapple fixtures, or add markers) or in current assets which include customer satellites without any addable interfaces to facilitate the RVD process. In this 
case the only information available is the satellite orbit determined by radar measurements [1]. This paper presents strategies for autonomous RVD taking into account a constellation of service satellites. The Lambert problem is solved to select the service satellite among the constellation with less $\Delta \mathrm{V}$ for a specific time of flight. A study case OOS scenario dealing with a non-cooperative satellite in an LEO orbit is presented. Moreover, the problem of estimating the relative pose between service and a non-cooperative satellite is investigated. It is considered a monocular vision system in order to recognize and inspect a non-cooperative customer satellite. We present a new formulation to identify the relative pose of the target by using only natural features of the target satellite (e.g. corners and edges). The presented algorithm is general and does not rely on markers mounted on the target as the case of many current assents in space.

Nowadays, integrated systems using technologies of autonomous navigation and machine vision, for inspection and rendezvous and docking operations are reaching the stage of real demonstration. In this line of work, we can cite the Demonstration of Autonomous Rendezvous Technology (DART) spacecraft launched in 2005 and the Japan's Experimental Test Satellite (ETS-VII). DART was designed to perform autonomous RVD with the MUBLCOM satellite. Due to anomalies with the craft's navigation system, the DART spacecraft has used more propellant than expected, aborting the mission [2]. The ETS-VII performed a successful RVD using a computer vision system. In that in-orbit test the docking operation used a ground teleoperated manipulator to dock the chaser spacecraft with a small target satellite [3]. A new service American spacecraft system with integrated systems of robotics, machine vision, and autonomous control for universal tugs is in development. That project is named spacecraft for the universal modification of orbits (SUMO) [4]. The tug system is being executed by the Naval Center for Space Technology (NCST) at the Naval Research Laboratory (NRL). That tug may become the first to rescue stranded satellites and extend the lifetime of costumer's satellites. Albert et al. analyzed spacecraft failures, suggesting that a rescue attempt would be commercially justified [4]. Several authors have been dealing with the estimation problem in the context of RVD. Diaz et al. have developed a Satellite Recognition and Inspection approach via Autonomous Navigation (RISANAR) test bed to simulated scenarios of RVD and also presented a satellite vision system based on pose estimation [5]. Cropp et al. have presented a method for estimating the relative position and orientation of a known target satellite by using only passive imagery [6]. Cropp et al. have used target images generated synthetically [7]. A cognitively-controlled vision system for recognition and tracking applied for RVD tasks is presented in $[8,9]$.

The paper is organized as follows: Section 2 shows a general description of the tasks of the service satellite. Section 3 summarizes the orbital maneuvers showing the algorithm to solve the Lambert problem for far maneuvers as well as the approach for close and inspection maneuvers using the Hill equations. Section 4 presents the pose estimation for point correspondence. Furthermore, shows the assumptions used and the dual quaternion parameterization for attitude and position. At the end of that section we present the pose error function associated with our problem. Section 5 reports on the results of orbital maneuvers and pose estimation results. The paper is concluded with a brief discussion and suggested future works.

\section{Reference mission scenario}

Before the vision system takes place, the service spacecraft performs transfer maneuvers to meet a point close to the target orbit and behind the target object. We define a hypothetical mission and show how such an analysis is performed in two different phases, far and proximity phases. The first phase we have performed far scale operations:

- The target is supposed to be in an altitude of $450 \mathrm{~km}$ above the Earth surface (LEO orbit). It was defined that the service satellite, or chaser satellite, will be placed around $300 \mathrm{~m}$ behind the target satellite, in the same orbit of the target (point M1 in Fig. 1. We perform a study taking into account a constellation of service satellites to choose the one that would have the best performance.

In the second phase, we have performed proximity scale operation (those at a distance less than $300 \mathrm{~m}$ from the target):

- The chaser vehicle is placed in an inspection orbit close to the target-first inspection point M2, shown in Fig. 1.

- A new maneuver in order to place the service satellite in another inspection orbit closer than that of the first inspection point M3 (see Fig. 1).

- Estimation of the relative position and orientation based on vision system.

- Final approach, straight line V-bar.

Fig. 1 shows the scenario of the servicing mission.

In the first phase, we have analyzed the time versus fuel consumption versus service satellite position. The second phase the estimation is made without target's landmarks but based on the own target structure and taking into account an assembly of points which fit the target features after image processing, e.g. segmentation. We are assuming that we already know the point features in the image plane which correspond to the points in the present model. The correspondence task is presented in reference [10].

\section{Orbital maneuvers}

\subsection{Rendezvous with the target vehicle: far maneuvers}

Initially, from the chaser vehicle initial orbit, a bi-impulsive maneuver must be performed to approach 


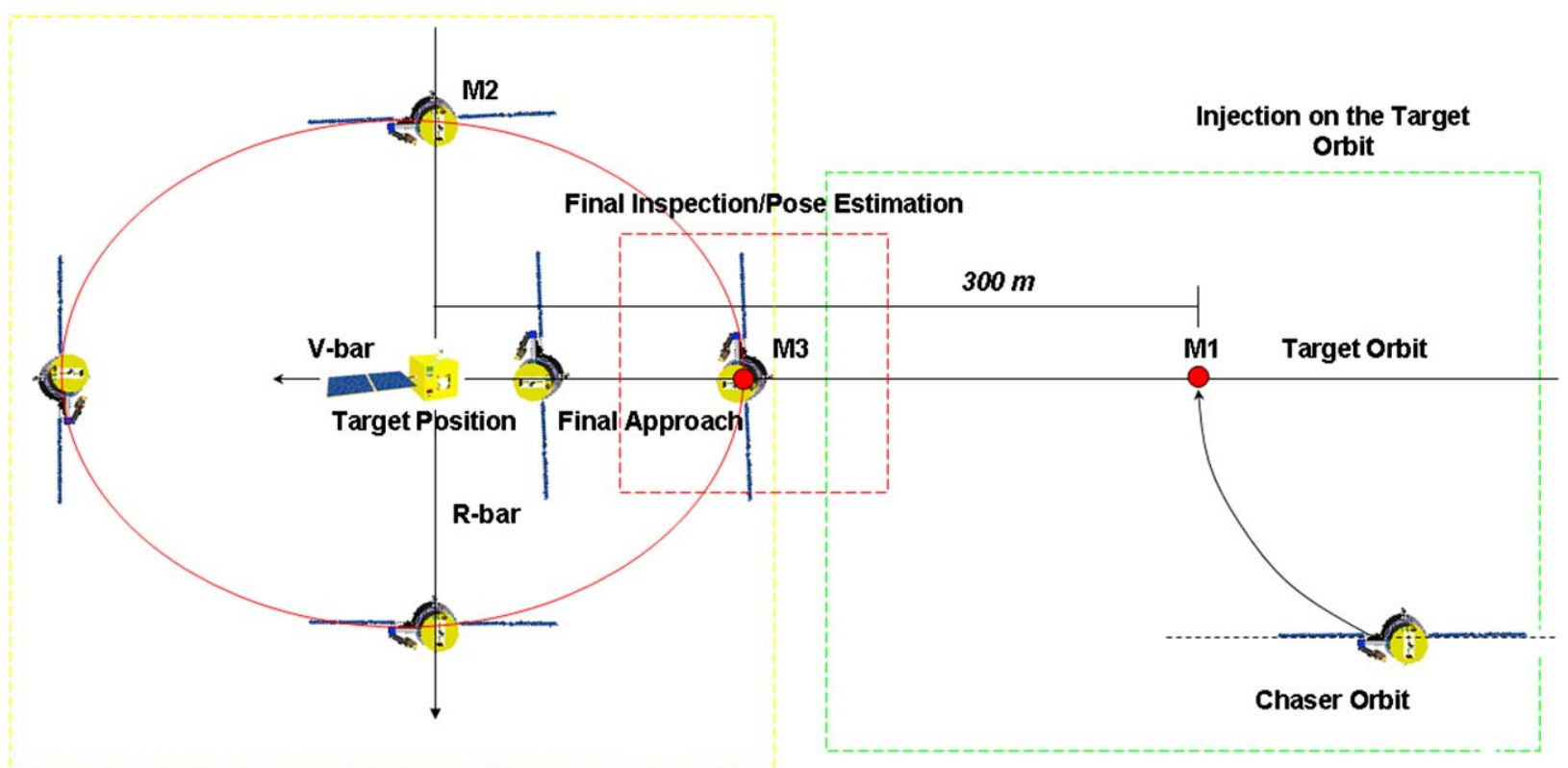

Fig. 1. Servicing mission scenario.

the target vehicle. In terms of implementation that operation requires the solution of the Lambert problem. This problem consists of calculating the transfer orbit that connects a point on the initial orbit (position of the chaser vehicle) to another point on the final orbit (position near to the target vehicle), in a given interval of time $t$.

The initial and final velocities in the transfer orbit are given by

$\vec{v}_{1}=\frac{\vec{r}_{2}-f(z) \vec{r}_{1}}{g(z)} ; \quad \vec{v}_{1}=\frac{\dot{g}(z) \vec{r}_{2}-\vec{r}_{1}}{g(z)}$

where $\vec{r}_{1}$ and $\vec{r}_{2}$ are the initial and final chaser positions, $\vec{v}_{1}$ and $\vec{v}_{2}$ are the initial and final chaser velocities. To solve the problem the functions $f(z)$ and $g(z)$ must be found. This problem is known as Gauss or Lambert problem and the applications are almost limitless and include satellite interception and rendezvous. A detailed study can be found in [11]. The algorithm to solve this problem via universal variables [12] can be described in the following way:

1. From the initial and the final position and considering the direction of the motion (long way or short way), evaluate the constant $A$ as

$A=\operatorname{sign}(\pi-\Delta \theta) \sqrt{\left|\vec{r}_{1}\right|\left|\vec{r}_{2}\right|(1+\cos \Delta \theta)}$

where $\Delta \theta$ is the transfer angle given by

$\Delta \theta=a \cos \left(\frac{\vec{r}_{1} \cdot \vec{r}_{2}}{\left|\vec{r}_{1}\right|\left|\vec{r}_{2}\right|}\right)$

$\operatorname{sign}(\pi-\Delta \theta)=-1 \Rightarrow \Delta \theta>\pi \Rightarrow$ long way otherwise short way.

2. Solve the constraint equation

$F(z)=x^{3}(z) S(z)+A \sqrt{y(z)}-t \sqrt{\mu}=0$ where

$S(z)=\frac{\sqrt{z}-\sin (\sqrt{z})}{\sqrt{z^{3}}} ; \quad C(z)=\frac{1-\cos (\sqrt{z})}{z}$

$y(z)=\left|\vec{r}_{1}\right|+\left|\vec{r}_{2}\right|-A \frac{1-z S(z)}{\sqrt{C(z)}} ; \quad x(z)=\sqrt{\frac{y(z)}{C(z)}}$

Pick a trial value for the universal variable $z$ in two stages: rough and fine search. The first stage consists in trial and error satisfying a rough tolerance. Then, the rough solution for $z$ is used as initial guess for the GaussNewton method in the second stage to find the fine solution.

3. When the method converges to a solution evaluate the functions $f(z), g(z)$ and $\dot{g}(z)$ :

$f(z)=1-\frac{y(z)}{\left|\vec{r}_{1}\right|}, \quad g(z)=A \sqrt{\frac{y(z)}{\mu}}, \quad \dot{g}(z)=1-\frac{y(z)}{\left|\vec{r}_{2}\right|}$

4. Compute the transfer velocities using Eq. (1).

5. By considering the transfer velocities and the velocities in the initial and final orbits calculate the necessary velocity increment as

$\Delta \vec{v}_{1}=\vec{v}_{1}-\vec{v}_{\text {initial }} ; \quad \Delta \vec{v}_{2}=\vec{v}_{\text {final }}-\vec{v}_{2}$

The state vectors in the initial and final of the transfer orbit can be obtained from the positions and velocities vectors. Once the state vectors are written the Keplerian elements of the transfer orbit are calculated [13].

\subsection{Rendezvous with the target vehicle: proximity maneuvers}

During the close and final approaches, the equations of the dynamics are written in the target local orbital frame. 
According to [14], the curvilinear orbit direction is approximated as a straight line and is usually named Vbar in the direction of the orbital velocity vector $\vec{V}$. The axis in the direction of the center of the Earth (radius vector $\vec{R}$ is named R-bar, (see Fig. 1 ), and the third axis completing the system of coordinates is named H-bar in the direction of the orbital angular momentum vector $\vec{H}$. For relative navigation, it becomes more convenient to keep the spacecraft as fixed points. For circular orbits, the relative motion between a target and chaser are the Hill equations

$\ddot{x}-2 \omega \dot{z}=f_{x}$

$\ddot{y}+\omega^{2} y=f_{y}$

$\ddot{z}+2 \omega \dot{x}-3 \omega^{2} z=f_{z}$

where $x, y$, and $z$ are the chaser position with respect to the target local orbital frame. The solution of those equations can be found in [4]. Based on that close form solution, we can study and design approaching maneuvers to inspect and execute a final approach. Some previous works have dealt with the inspection phase $[5,6]$. Soon after the injection of the chaser in the target orbit (assuming $300 \mathrm{~m}$ behind the target) we obtain, based on the solution of the Hill equations, an in-plane guidance function for inspection and final approach. The calculate thrust profile is show in Fig. 2 . The thrust profile aims to obtain an orbital trajectory which suits the reference scenario Fig. 1. At the point M1 (see Fig. 1), we carry out the first close orbital maneuver by a continuous impulse until M2. The goal of the first close maneuver is to put the service vehicle in the first inspection orbit. During the first inspection phase it takes place the visual system. Eventually, at this point some adjustment could be made in order to correct the position of the chaser with respect to the target. After a few orbits, the chaser is supposed to have executed the second maneuver that put the service spacecraft closer to the target in another orbit (point M3) also for inspection. At this point, the visual system would manage to obtain a more accurate estimation of the target position and attitude (pose). After accomplishing the estimation in the inspection phase the chaser approaches to the target along V-bar direction.

\section{Pose estimation: point correspondence}

During the inspection phase the autonomous vision system has to perform the pose estimation. By estimating the position and orientation of the target, the chaser would execute a reliable final approach and docking. In order to state the pose estimation algorithm, let $\mathbf{M}_{i}$ be an elemental point described in the coordinates of the frame $X_{i}^{t}, Y_{i}^{t}$, and $Z_{i}^{t}$, as shown in Fig. 3. Consider that the object model is represented by a set of points $\mathbf{M}_{i}$, and their projection coordinates $x_{i}$ and $y_{i}$ in the image frame as shown in the Fig. 3 (where only $\mathbf{m}_{i}$ projection of $\mathbf{M}_{i}$ are shown). Assuming a pinhole camera model, the collinearity between $\mathbf{M}_{i}$ and $\mathbf{m}_{i}$ and the center of projection $O$ is expressed by the equations [15]:

$x_{i}=\frac{f X_{i}^{C}}{f-Z_{i}^{C}} ; \quad y_{i}=\frac{f Y_{i}^{C}}{f-Z_{i}^{C}}$

where $f$ is the focal length of the camera the distance from the lens to the image plane. The homogeneous transformation of the Cartesian coordinates $\left(X^{C}, Y^{C}, Z^{C}\right)$ into the homogeneous coordinates $\left(k X^{C}, k Y^{C}, k Z^{C}, k\right)(k$ is a

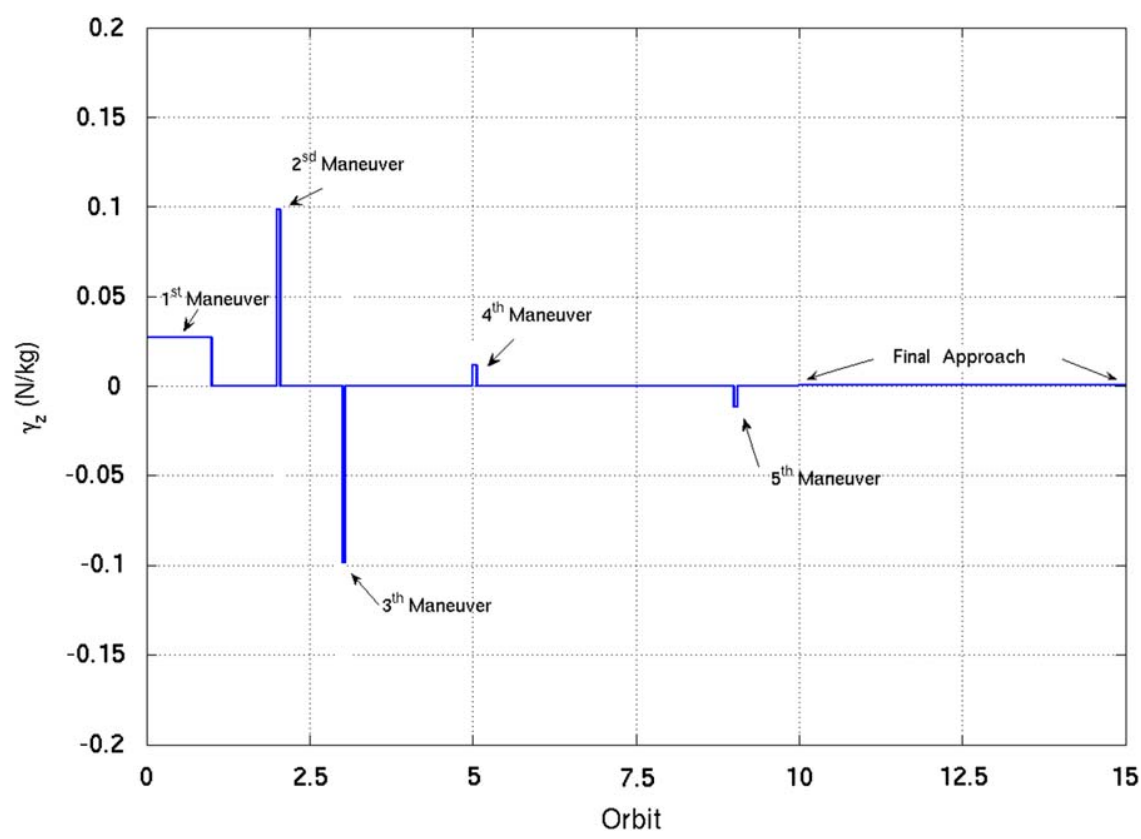

Fig. 2. Thruster chaser profile for close maneuvers: inspection and final approach phase. 


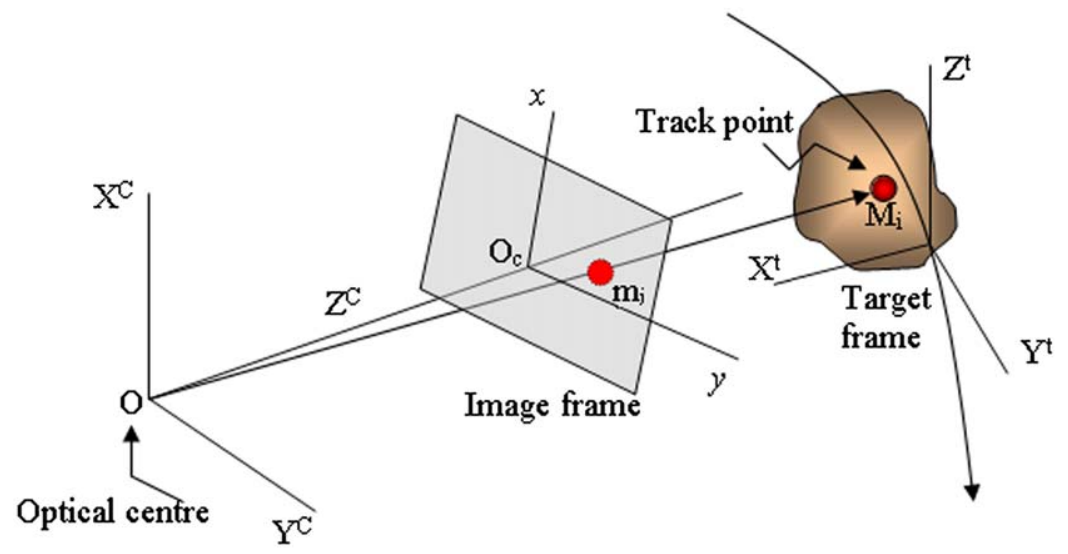

Camera frame

Fig. 3. Target, camera, and image coordinate frames.

normalization factor) is given by the perspective matrix:

$\mathbf{P}=\left[\begin{array}{cccc}1 & 0 & 0 & 0 \\ 0 & 1 & 0 & 0 \\ 0 & 0 & 1 & 0 \\ 0 & 0 & -\frac{1}{f} & 1\end{array}\right]$

The perspective transformation which relates the homogeneous coordinates to the homogeneous image coordinates is defined as

$\left[\begin{array}{c}k X^{C} \\ k Y^{C} \\ k Z^{C} \\ k-\frac{k Z^{C}}{f}\end{array}\right]=\mathbf{P}\left[\begin{array}{c}k X^{C} \\ k Y^{C} \\ k Z^{C} \\ k\end{array}\right]$

We can obtain Eq. (12) from Eq. (14) just dividing it by the fourth vector component. In order to represent the camera coordinates with respect to target coordinates $\left(k X^{t}, k Y^{t}, k Z^{t}\right)$ we use the transformation as follows:

$\left[\begin{array}{c}k X^{C} \\ k Y^{C} \\ k Z^{C} \\ k-\frac{k Z^{C}}{f}\end{array}\right]=\mathbf{P E I}\left[\begin{array}{c}k X^{t} \\ k Y^{t} \\ k Z^{t} \\ k\end{array}\right]$

where $\mathbf{E}$ and $\mathbf{I}$ are matrices $4 \times 4$ representing the rigid transformations from target frame to camera frame, respectively. Those matrices are established during onground calibration. On-ground calibration shall provide the intrinsic parameters (e.g. focal length $f$ ) of the camera and the extrinsic parameters (e.g. matrices $\mathbf{E}$ and $\mathbf{I}$ ) as well. Once the camera calibration is accomplished $\mathbf{E}$ and $\mathbf{I}$ matrices are given. From Eq. (15), we can write the camera model as

$x_{i}=\frac{a_{11} X_{i}^{t}+a_{12} Y_{i}^{t}+a_{13} Z_{i}^{t}+a_{14}}{a_{41} X_{i}^{t}+a_{42} Y_{i}^{t}+a_{43} Z_{i}^{t}+a_{44}}$

$y_{i}=\frac{a_{21} X_{i}^{t}+a_{22} Y_{i}^{t}+a_{23} Z_{i}^{t}+a_{24}}{a_{41} X_{i}^{t}+a_{42} Y_{i}^{t}+a_{43} Z_{i}^{t}+a_{44}}$ where $\left(x_{i}, y_{i}\right)$ are 2D coordinates of the projected 3D object in the image frame, $a_{i j},(i=1, \ldots, 3, j=1, \ldots, 4)$ (components of $\mathbf{A}=\mathbf{P E I}$ ) are the camera intrinsic and extrinsic parameters that describe the transformation of any point from the target to the camera coordinate frame. Eqs. (16) and (17) can be written more compactly as

$\left[\begin{array}{lll}1 & 0 & -x_{i}\end{array}\right] A^{*}\left[\begin{array}{c}X_{i}^{t} \\ Y_{i}^{t} \\ Z_{i}^{t}\end{array}\right]+\left[\begin{array}{lll}1 & 0 & -x_{i}\end{array}\right]\left[\begin{array}{l}a_{14} \\ a_{24} \\ a_{44}\end{array}\right]=0$

$\left[\begin{array}{lll}1 & 0 & -y_{i}\end{array}\right] A^{*}\left[\begin{array}{c}X_{i}^{t} \\ Y_{i}^{t} \\ Z_{i}^{t}\end{array}\right]+\left[\begin{array}{lll}0 & 1 & -y_{i}\end{array}\right]\left[\begin{array}{c}a_{14} \\ a_{24} \\ a_{44}\end{array}\right]=0$

where matrix $A^{*}$ is defined in terms of matrix $A$ as

$A^{*}=\left[\begin{array}{lll}a_{11} & a_{12} & a_{13} \\ a_{21} & a_{22} & a_{23} \\ a_{41} & a_{42} & a_{43}\end{array}\right]$

All elements of the matrix $A^{*}$ are known. Those elements were obtained by a calibration process $[16,17]$. That matrix is used to take simulate pictures of the target object.

In order to establish a new rigid transformation let us consider the model coordinate frame defined $\operatorname{as}\left(X^{M}, Y^{M}, Z^{M}\right)$. The rigid transformation from the model to target coordinate frame is given by

$\left[\begin{array}{c}X_{i}^{t} \\ Y_{i}^{t} \\ Z_{i}^{t}\end{array}\right]=\mathbf{R}\left[\begin{array}{c}X_{i}^{M} \\ Y_{i}^{M} \\ Z_{i}^{M}\end{array}\right]+\mathbf{T}$

where $\mathbf{R}$ is a rotation matrix and $\mathbf{T}$ is a translation vector. $X_{i}^{M}, Y_{i}^{M}$, and $Z_{i}^{M}$ are coordinates of the same points with respect to the model reference frame.

The pose estimation task is stated as: find the rigid transformation, the rotation matrix $\mathbf{R}$ and the translation vector $\mathbf{T}$, which lead to a best fitting between the object model and the obtained image data.

In order to represent simultaneously the rotations and translations in a unique formulation, it is convenient to 
write the pose equations by using the dual quaternion. In order to accomplish this, consider some mathematical manipulations as follows:

First we rewrite Eqs. (18) and (19) as

$\left[\begin{array}{lll}a_{11}-a_{41} x_{i} & a_{12}-a_{42} x_{i} & a_{13}-a_{43} x_{i}\end{array}\right]\left[\begin{array}{c}X_{i}^{t} \\ Y_{i}^{t} \\ Z_{i}^{t}\end{array}\right]+a_{14}-a_{44} x_{i}=0$

$\left[\begin{array}{lll}a_{21}-a_{41} \dot{y}_{i} & a_{22}-a_{42} x_{i} & a_{23}-a_{43} y_{i}\end{array}\right]\left[\begin{array}{c}X_{i}^{t} \\ Y_{i}^{t} \\ Z_{i}^{t}\end{array}\right]+a_{24}-a_{44} y_{i}=0$

Then we redefine new vectors and constants as

$\mathbf{v}_{x_{i}}=\left[\begin{array}{lll}a_{11}-a_{41} x_{i} & a_{12}-a_{42} x_{i} & a_{13}-a_{43} x_{i}\end{array}\right]^{T}$

$\mathbf{v}_{y_{i}}=\left[\begin{array}{lll}a_{21}-a_{41} \dot{y}_{i} & a_{22}-a_{42} x_{i} & a_{23}-a_{43} y_{i}\end{array}\right]^{T}$

$\delta_{x_{i}}=a_{14}-a_{44} x_{i} ; \quad \delta_{y_{i}}=a_{24}-a_{44} y_{i}$

Finally, we rewrite Eqs. (18) and (19) as

$\mathbf{v}_{x_{i}}\left[\begin{array}{c}X_{i}^{t} \\ Y_{i}^{t} \\ Z_{i}^{t}\end{array}\right]+\delta_{x_{i}}=0 ; \quad \mathbf{v}_{y_{i}}\left[\begin{array}{c}X_{i}^{t} \\ Y_{i}^{t} \\ Z_{i}^{t}\end{array}\right]+\delta_{y_{i}}=0$

By substituting Eq. (21) into Eq. (27) we have

$\mathbf{v}_{x_{i}}\left(\mathbf{R}\left[\begin{array}{c}X_{i}^{M} \\ Y_{i}^{M} \\ Z_{i}^{M}\end{array}\right]+\mathbf{T}\right)+\delta_{x_{i}}=0$

$\mathbf{v}_{y_{i}}\left(\mathbf{R}\left[\begin{array}{c}X_{i}^{M} \\ Y_{i}^{M} \\ Z_{i}^{M}\end{array}\right]+\mathbf{T}\right)+\delta_{y_{i}}=0$

The vectors $\mathbf{v}_{x_{i}}, \mathbf{v}_{y_{i}}$ and $\mathbf{X}_{i}=\left(X_{i}^{M}, Y_{i}^{M}, Z_{i}^{M}\right)$ represented as dual vectors are given by the following matrices:

$\mathbf{u}_{x_{i}}=\left[\begin{array}{c}0 \\ \mathbf{v}_{x_{i}}\end{array}\right], \quad \mathbf{u}_{y_{i}}=\left[\begin{array}{c}0 \\ \mathbf{v}_{y_{i}}\end{array}\right], \quad \mathbf{M}_{i}^{d}=\left[\begin{array}{c}0 \\ \mathbf{X}_{i}\end{array}\right]$

\subsection{Screw transformation: dual quaternions}

We can describe the rotation and translation in a unified notation by using a unit dual quaternion. The interesting feature of parameterizing screw transformation or rigid transformations with dual quaternion resides in the simple involved algebraic manipulations. In addition, this parametrization allows us to describe pose problems in a more simple, easy and elegant form than those other forms of parametrization for rigid transformations $[18,19]$. Formally, a screw transformation is defined by the triplet $(d, \phi, \Gamma$,), where $d$ is the length of the translation along the screwing axis, $\phi$ the rotation angle around the screwing axis, $\Gamma$ the screwing axis which may be represented parametrically by a direction unit vector $\hat{\mathbf{r}}$ and a position vector $\hat{\mathbf{l}}$, such that $\hat{\mathbf{r}} \cdot \hat{\mathbf{i}}=0$. We represent a dual quaternion as $\mathbf{q}=\mathbf{r}+\varepsilon \mathbf{s}$, where $\mathbf{q}$ represents a rigid transformation. Its real $\mathbf{r}$ and dual part $\mathbf{s}$ are defined by

$\mathbf{r}=\left(\cos \frac{\phi}{2}, \sin \frac{\phi}{2} \hat{\mathbf{r}}\right) ; \quad \mathbf{s}=\left(-\frac{d}{2} \sin \frac{\phi}{2}, \frac{d}{2} \cos \frac{\phi}{2} \hat{\mathbf{r}}+\sin \frac{\phi}{2} \hat{\mathbf{r}} \times \hat{\mathbf{i}}\right)$

Fig. 4 shows a screw representation of a free flight satellite. Chasle's theorem states that such screw transformation can describe any rigid transformation [20].

It is possible to verify that the following two constraints hold:

$\mathbf{r} \cdot \mathbf{r}=1 ; \quad \mathbf{r} \cdot \mathbf{s}=0$

The rotation matrix $\mathbf{R}$ and the translation vector $\mathbf{T}$ can be derived by using the following equations:

$\left[\begin{array}{cc}1 & \mathbf{0}_{1 \times 3} \\ \mathbf{0}_{3 \times 1} & \mathbf{R}\end{array}\right]=W(\mathbf{r})^{T} Q(\mathbf{r}) ; \quad\left[\begin{array}{l}0 \\ \mathbf{T}\end{array}\right]=2 W(\mathbf{s})^{T}$

where $W(\mathbf{r})$ and $Q(\mathbf{r})$ are $4 \times 4$ matrices associated with the real part of the unit dual quaternion $\mathbf{r}$ :

$Q(\mathbf{r})=\left[\begin{array}{cccc}r_{0} & -r_{1} & -r_{2} & -r_{3} \\ r_{1} & r_{0} & -r_{3} & r_{2} \\ r_{2} & r_{3} & r_{0} & -r_{1} \\ r_{3} & -r_{2} & r_{1} & r_{0}\end{array}\right] ; \quad W(\mathbf{r})=\left[\begin{array}{cccc}r_{0} & -r_{1} & -r_{2} & -r_{3} \\ r_{1} & r_{0} & r_{3} & -r_{2} \\ r_{2} & -r_{3} & r_{0} & r_{1} \\ r_{3} & r_{2} & -r_{1} & r_{0}\end{array}\right]$

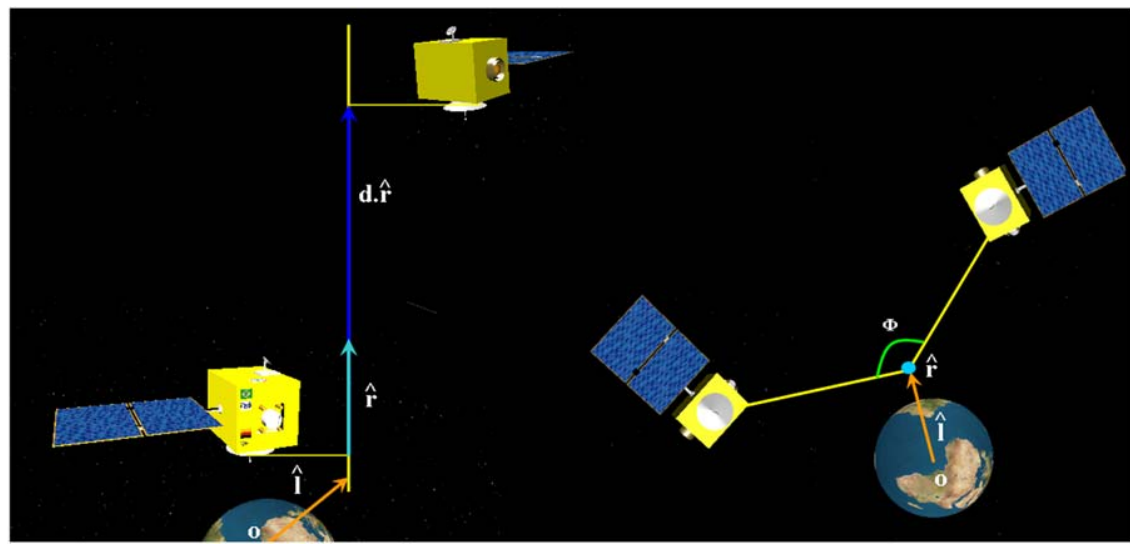

Fig. 4. Screw satellite motion, side and top views. The rigid transformation is a screw: rotation about an axis by angle $\Phi=2 \phi$ and a translation of magnitude $d$ along the $\Gamma$ axis in the direction determined by a unit vector $\hat{\mathbf{r}}$. 


\subsection{Pose equations}

At this point, we can compute the pose constraint equations by substituting Eqs. (30) and (34) into Eqs. (28) and (29) and rearranging we obtain

$\mathbf{u}_{x_{i}}^{T} W(\mathbf{r})^{T} Q(\mathbf{r}) \mathbf{M}_{i}^{d}+\mathbf{u}_{x_{i}}^{T} 2 W(\mathbf{r})^{T} \mathbf{s}+\delta_{x_{i}}=0$

$\mathbf{u}_{y_{i}}^{T} W(\mathbf{r})^{T} Q(\mathbf{r}) \mathbf{M}_{i}^{d}+u_{y_{i}}^{T} 2 W(\mathbf{r})^{T} \mathbf{s}+\delta_{y_{i}}=0$

By using the following property $Q(\mathbf{a}) \mathbf{b}=W(\mathbf{b}) \mathbf{a}$ in conjunction with the Eq. (35), we finally obtain the first pose constraint equation as

$\mathbf{r}^{T} Q\left(\mathbf{u}_{x_{i}}\right)^{T} W\left(\mathbf{M}_{i}^{d}\right) \mathbf{r}+2 \mathbf{r}^{T} Q\left(\mathbf{u}_{x_{i}}\right)^{T} \mathbf{s}+\delta_{x_{i}}=0$

Similarly, we can obtain the expression for the second pose constraint equation as

$\mathbf{r}^{T} Q\left(\mathbf{u}_{y_{i}}\right)^{T} W\left(\mathbf{M}_{i}^{d}\right) \mathbf{r}+2 \mathbf{r}^{T} Q\left(\mathbf{u}_{y_{i}}\right)^{T} \mathbf{s}+\delta_{y_{i}}=0$

It is worth to note that in Eqs. (37) and (38), the inputs are $\mathbf{u}_{x_{i}}$ coordinates of the points $i=1, \ldots, n$ in the image along $x ; \mathbf{u}_{y_{i}}$ coordinates of the points $i=1, \ldots, n$ in the image along $y$, and $\mathbf{M}_{i}^{d}$ coordinates $\left(X_{i}^{M}, Y_{i}^{M}, Z_{i}^{M}\right)$ of the points $i=1, \ldots, n$ of the object model. The obtained outputs are $\mathbf{r}$ (the real part of the unit quaternion), and $\mathbf{s}$ (the dual part of the dual quaternion).

\subsection{Error function}

Based on the constraint Eqs. (37) and (38), we can write a new expression that represents the error function associated with our problem. It can be shown that in the case of point correspondence the error function to be minimized is

$F(\mathbf{r}, \mathbf{s})=\sum_{i=1}^{n}\left[\mathbf{r}^{T} Q^{T} W \mathbf{r}+2 \mathbf{r}^{T} Q^{T} \mathbf{s}+\delta_{x_{i}}\right]^{2}+\left[\mathbf{r}^{T} Q^{T} W \mathbf{r}+2 \mathbf{r}^{T} Q^{T} \mathbf{s}+\delta_{y_{i}}\right]^{2}$

plus the constraint Eq. (32) multiplied by the Lagrange multipliers

$C_{1}=(\mathbf{r} \cdot \mathbf{r}-1)^{2} ; \quad C_{2}=(\mathbf{r} \cdot \mathbf{s})^{2}$

It is now clear that the minimization of the error function is equivalent to the solution of the non-linear least square problem given by the minimization of the function

$S(\mathbf{x})=\sum_{j=1}^{m} \Theta_{j}^{2}(\mathbf{x})$

where $S(\mathbf{x})$ is the extend function to be minimized, such that $\mathbf{x} \in \mathfrak{R}^{n}$. In our problem, we have $n=8$, from which 4 are the real part $\mathbf{r}$ and 4 are the dual part $\mathbf{s}$. Here, $m=2 p+2$ where $p$ is the number of correspondence points. To solve the quadratic error function we have used and compared the following numerical methods for solution of non-linear least square problems: Newton method [21], Gauss-Newton method, and LevenbergMarquardt method [22,23].

\section{Simulations and results}

\subsection{Far maneuvers}

In this work, it was considered a constellation of 8 service satellites in a circular equatorial parking orbit at an altitude of $450 \mathrm{~km}$. The service satellites were

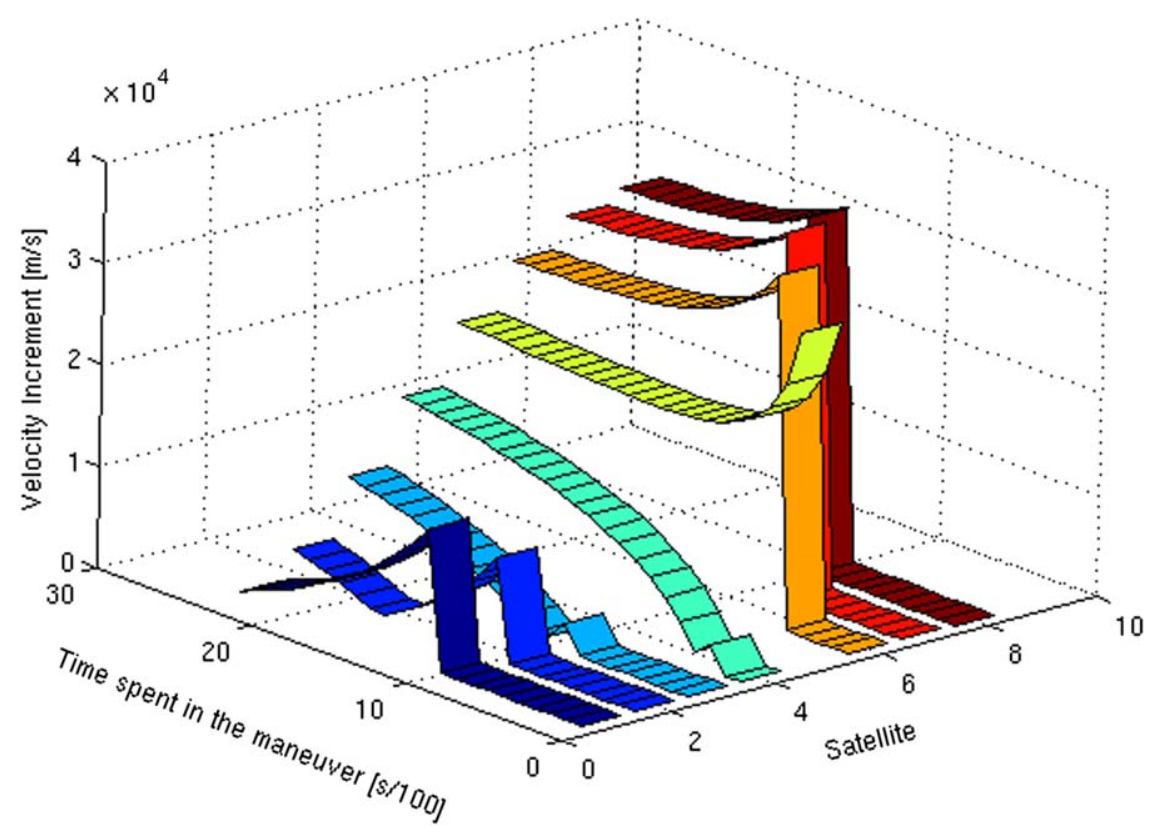

Fig. 5. Total velocity increment for the short way maneuvers. 


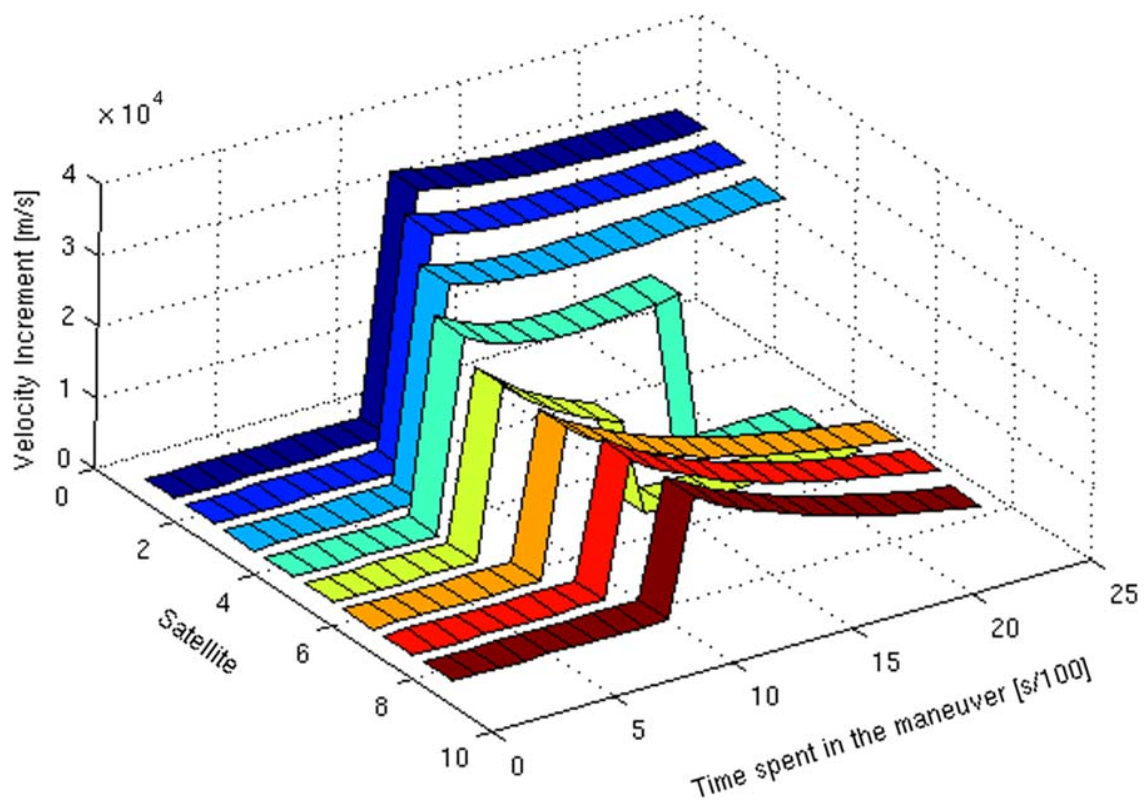

Fig. 6. Total velocity increment for the long way maneuvers.

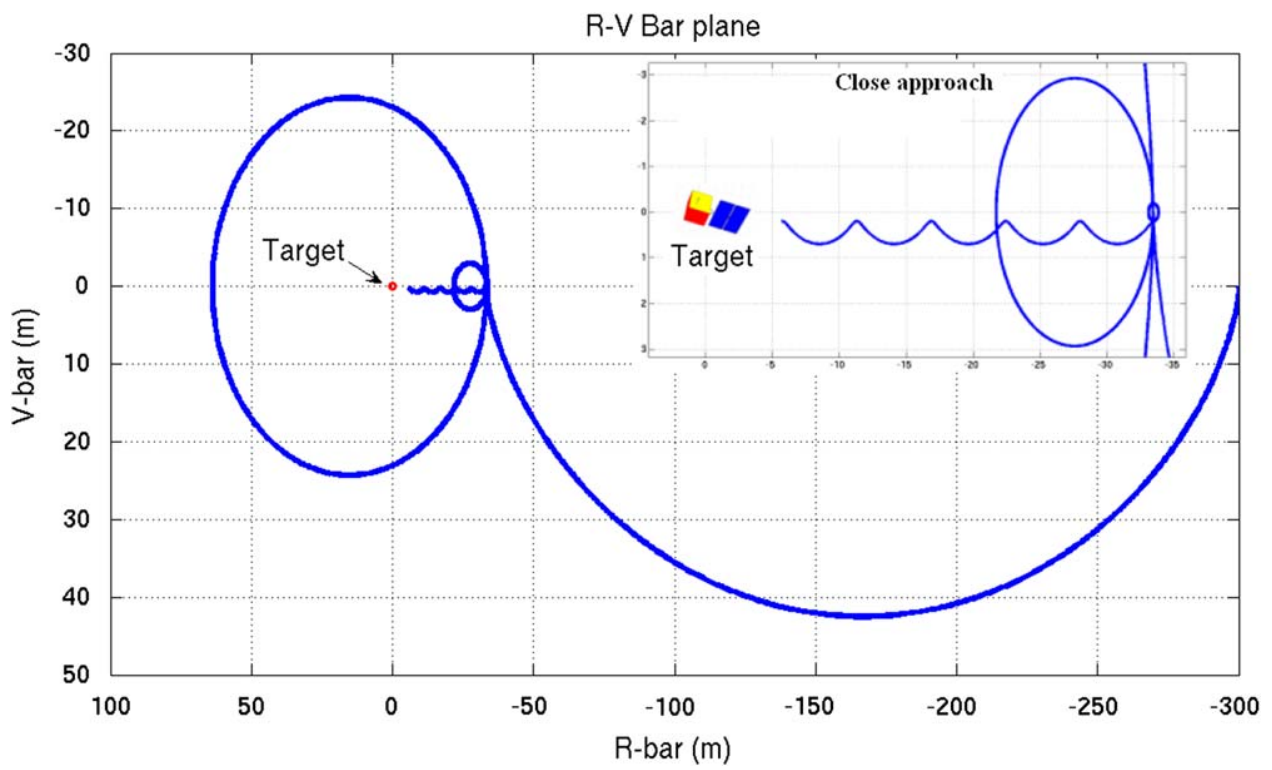

Fig. 7. Trajectory of the vehicle chaser during close maneuvers.

positioned in that orbit in a configuration of $45^{\circ}$ separated from each other. In order to accomplish the far maneuver, it is necessary to have some knowledge of the target object orbit so that the chaser orbital transference could be done correctly. The mean anomaly of the position near to the target vehicle, in the final orbit, was considered as $165^{\circ}$. Based on that assumption a set of transfer maneuvers was calculated for each satellite using the Lambert problem model [12]. The time spent to perform the bi-impulsive maneuver was set from 400 to $2700 \mathrm{~s}$. Therefore, 416 maneuvers were calculated considering the long way $(\Delta \theta>\pi)$ and the short way $(\Delta \theta<\pi)$. The
Table 1

True screw parameters.

\begin{tabular}{ll}
\hline$\phi$ & $47^{\circ}$ \\
$\mathbf{L}$ & {$[111]$} \\
$d$ & 2.3094 \\
\hline
\end{tabular}

velocity increments of these maneuvers are shown in Figs. 5 and 6.

To choose the best maneuver a multi-objective optimization study must be done. It can be desired to minimize simultaneously, the fuel consumption (total 
velocity increment) and the time spent in the maneuvers. However, those objectives, generally, are conflicting. When one of them is optimized the other may be not. In this case, it is practically impossible to determine the optimal solution that minimizes both objectives simultaneously. But, when one of the objectives is fixed the other is easily optimized and the solution can be determined.

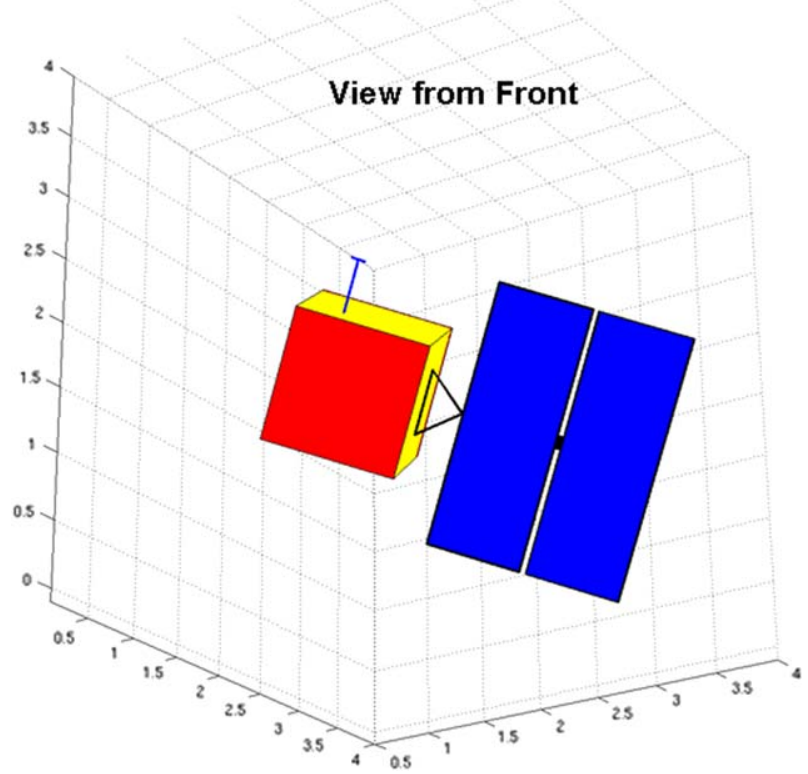

It is worth to say that the multi-objective optimization is not a main goal of this work. A survey of multi-objective optimization methods can be found in [24,25]. In [26,27], the application of a new multi-objective method was used to find the best orbital maneuver from a set of maneuvers previously calculated. The application of this new method could be used in this work to choose which service satellite should be maneuvered and which maneuver

Fig. 8. Simulated target object.
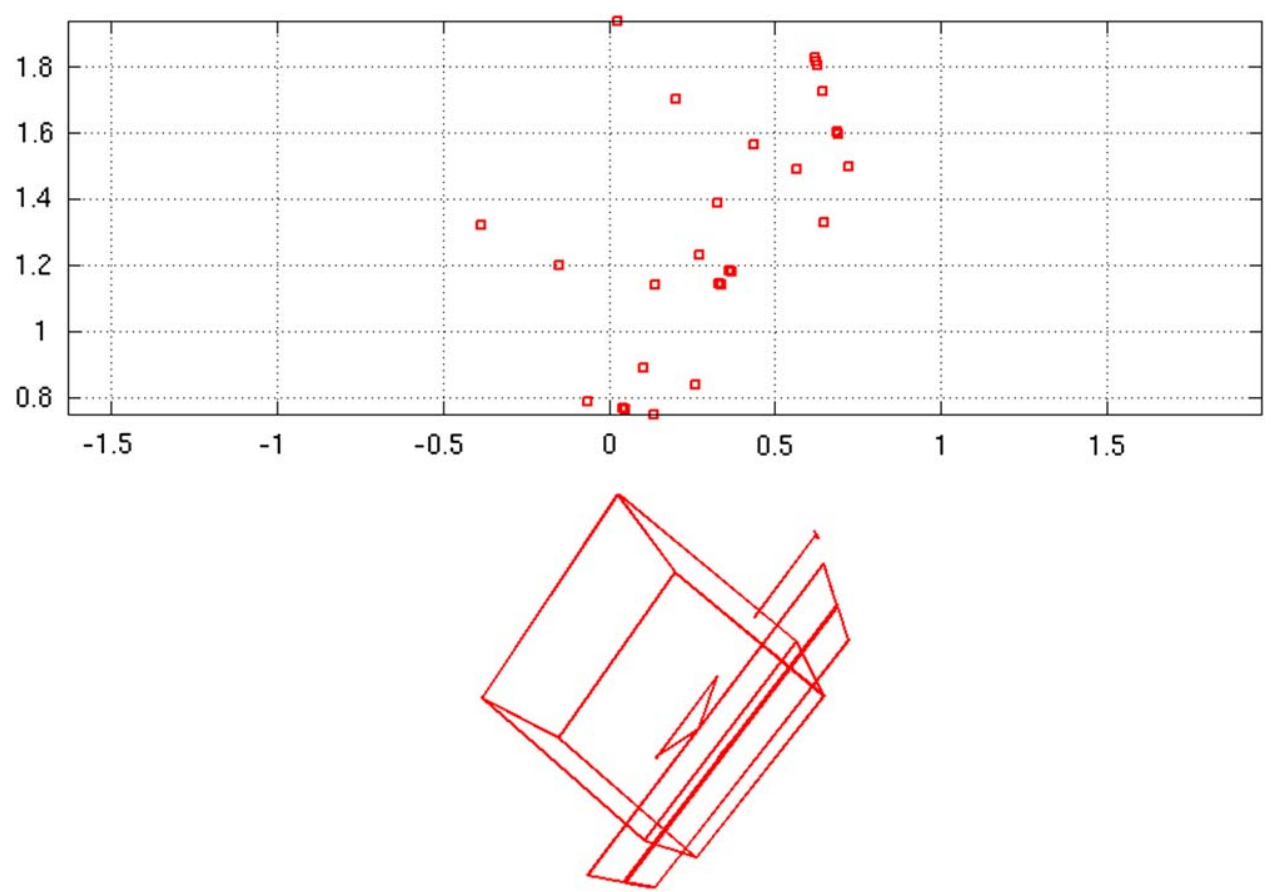

Fig. 9. Projected points ( 36 red points), and simulated target image (only lines) (for interpretation of the references to color in this figure legend, the reader is referred to the web version of this article). 
should be applied. However, the optimization approach is beyond the scope of this work.

\subsection{Close maneuvers: inspection and final approaching}

The trajectory of the service satellite during the first and the second inspections is shown in Fig. 7 (blue trajectory). Note that both inspection trajectories are circumferences. The large one illustrates a flight around the target; this sort of trajectory shall be used to achieve proper light conditions during visual inspection. The small circumference inspection illustrates the case where the chaser is kept behind the target. This one shall estimate

Table 2

Pose performance for different methods.

\begin{tabular}{llll}
\hline & N. of iterations & Residual & CPU time $(\mathrm{s})$ \\
\hline LM & 16 & $10^{-30}$ & 4.89 \\
GN & 9 & $10^{-30}$ & 2.76 \\
N & 88 & $10^{-14}$ & 20.95 \\
\hline
\end{tabular}
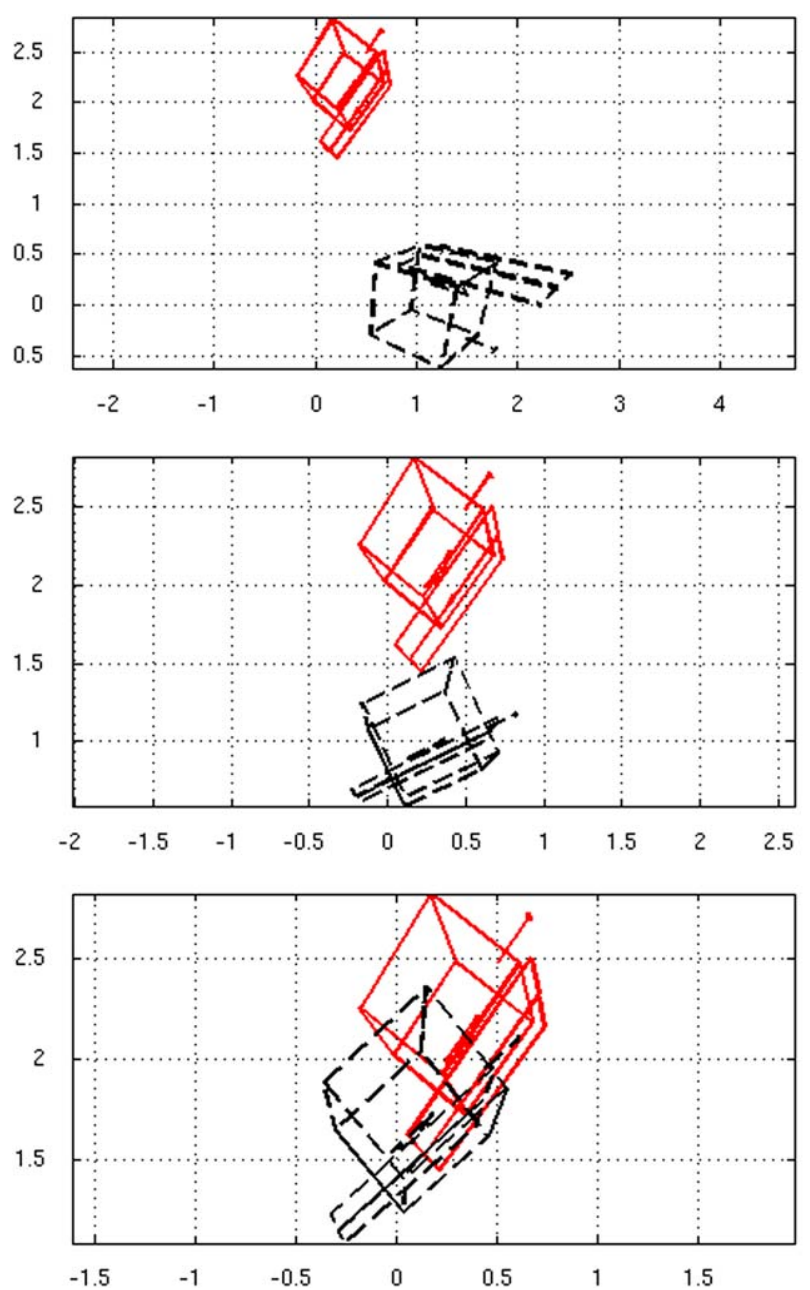

accurately the target's pose and eventually be used to make corrections in the position of the service satellite before the final approach. The final approach is shown by enlarging Fig. 7. The last maneuver is done after few orbits of the target. In fact that trajectory has a sinusoidal form with amplitude around centimeters and frequency equal to the inverse of the orbital period of the target.

\subsection{Pose estimation results}

In order to test the pose algorithm, random values for orientation and position of the satellite were taken. The screw elements are shown in Table 1 . We have used a simple target object to carry out our analysis and compare the optimization methods.

The target was simulated as a simple 3D object (see Fig. 8). The position of this target in space as viewed from front and top sides is shown in Fig. 8.

By applying the pinhole camera model we obtain the simulated image [15]. Fig. 9 shows the points of the target
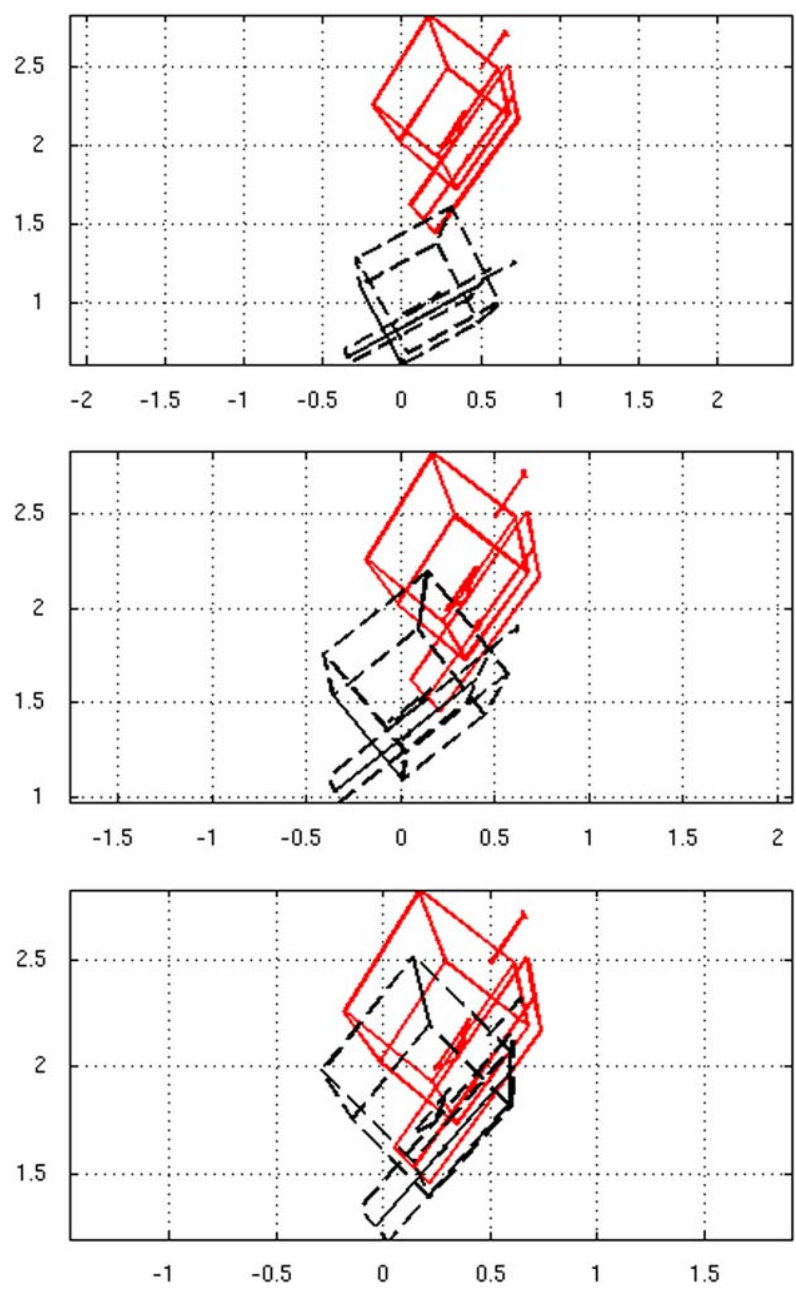

Fig. 10. Pose estimation process using the Newton method. 

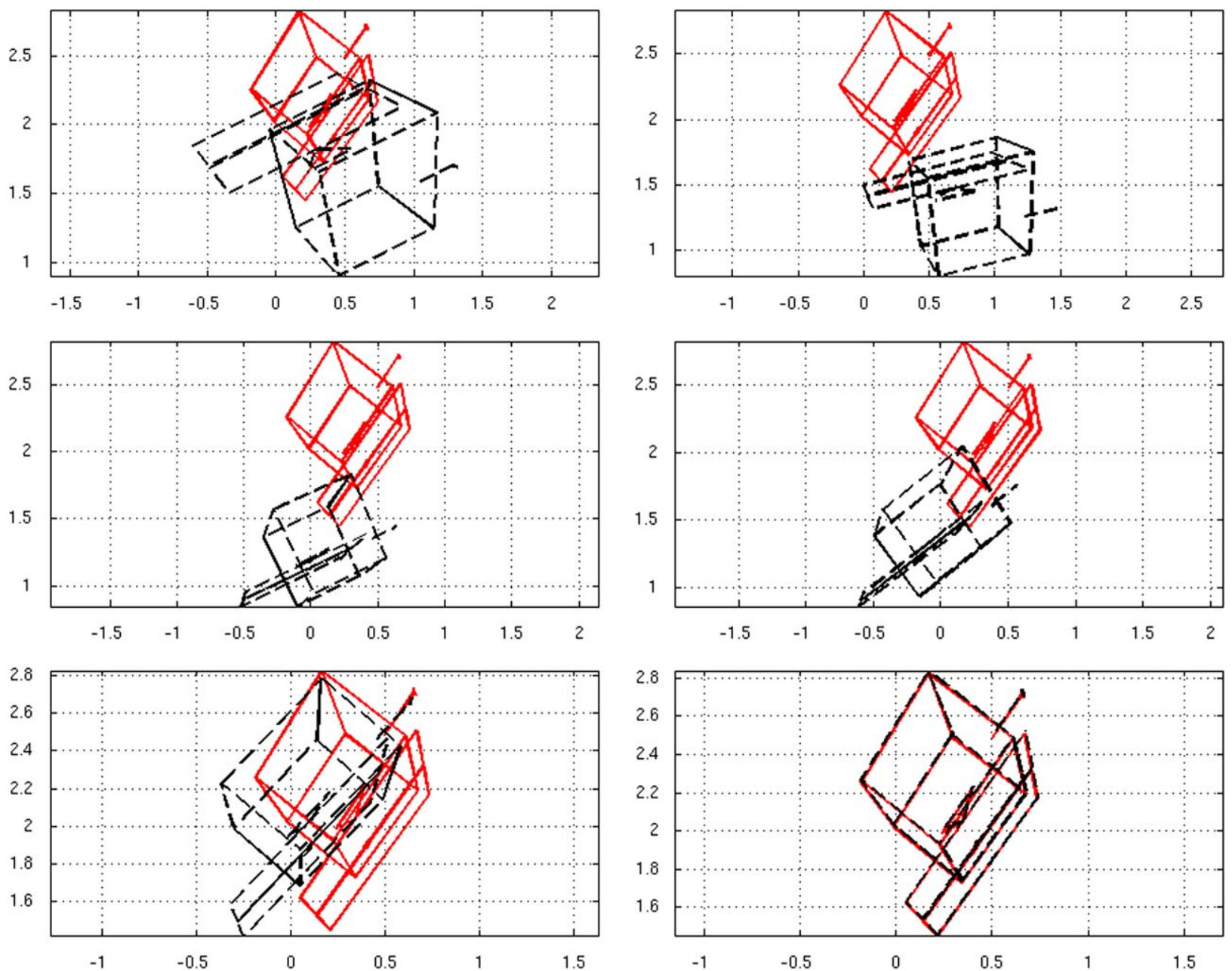

Fig. 11. Pose estimation process using the Levenberg-Marquardt method.

and simulated image in the actual position according to the above screw transformation shown in Table 1 . The assumed target model is formed by 36 points and the pose correspondence task is not considerate.

Table 2 shows the performance of the pose algorithm using different numerical methods such as LevenbergMarquardt (LM), Gauss Newton (GN), and Newton (N). The initial guess used for all methods was settled as $\mathbf{q}_{0}=\left[\begin{array}{llllllll}1 & 0.5 & 0.5 & 0.5 & 0.5 & 0.5 & 0.5 & 0.5\end{array}\right]^{T}$

The next figures show the pose estimation process using Newton, Levenberg-Marquardt, and Gauss-Newton methods. The red lines represent the image and the black dotted line represents the projection of the model after the iteration. The iteration sequence $1,3,5,7,9$, and 10 are shown in Figs. 10-12. We see that the pose estimation using the Gauss-Newton method presents a better performance in terms of time compared with the Newton and Levenberg-Marquardt methods. LM and GN methods achieve the same accuracy, but the GN converges faster. It relies on the fact that GN method is generally more effective than the LM method when the residual is zero at the solution.

\section{Conclusions and suggestion for future works}

In this work, a methodology applied during the far and close service satellite operations was presented. In the far operations, the Lambert problem was solved in two computed search stages (rough and fine search), achieving accurate results in a reduced CPU time. Those results allow us to choose the best service satellite to be maneuvered and the best maneuver to be implemented, considering the flight time and the fuel consumption. However, to make this choice autonomously a multi-objective criterion must be used. The close RVD operations were divided into inspection and final approach phases. In the inspection phase, we presented a scenario two inspection orbits in such a way that the service satellite could autonomously compute the pose of the target. The pose algorithm was developed aiming its integration into the vision and navigation systems for future applications in real systems. Although the analysis was conducted assuming the observation of all characteristic point of the target, the algorithm still can handle with the situation where some 

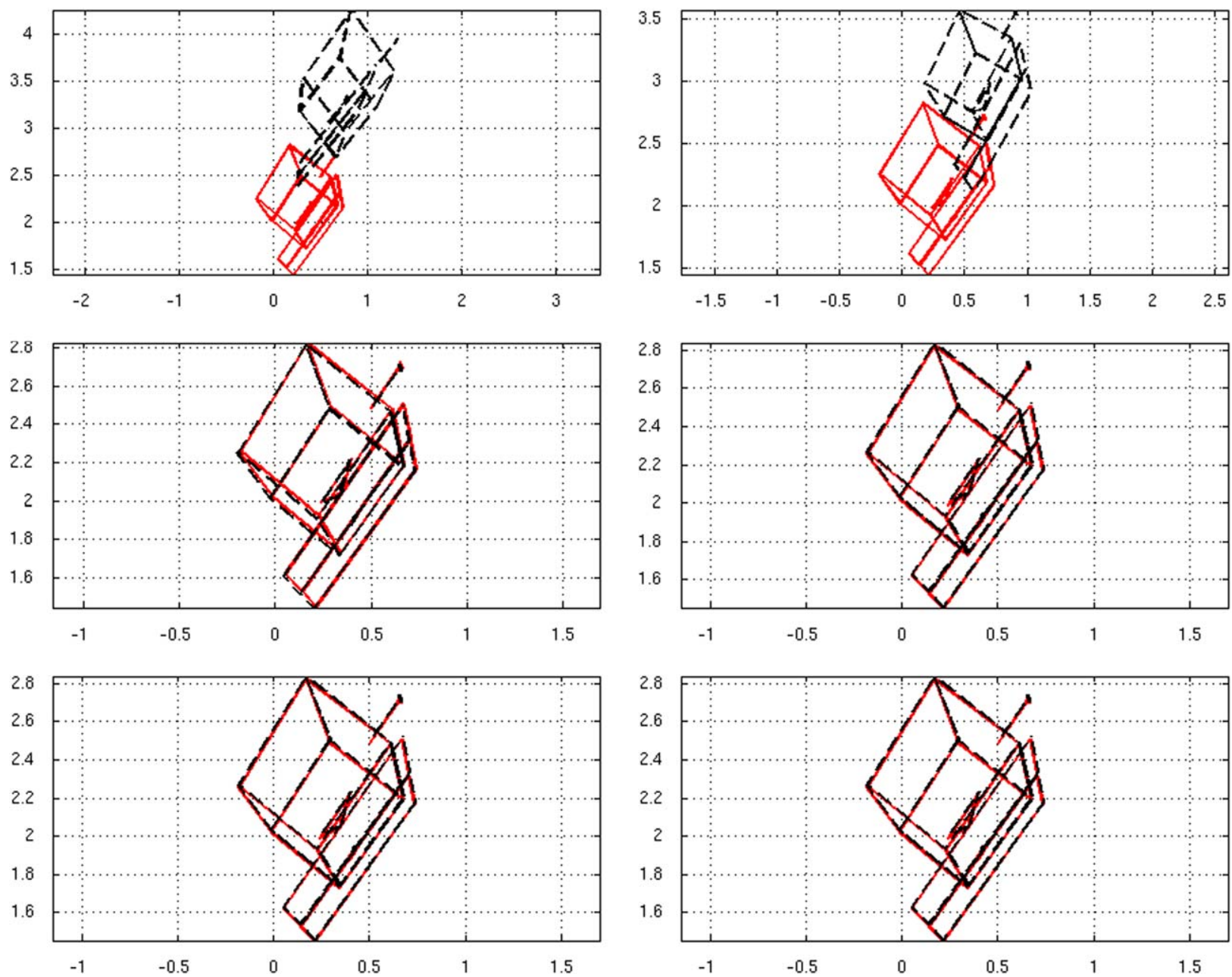

Fig. 12. Pose estimation process using the Gauss-Newton method.

points are hidden by the body of the target. In such case, at least 3 points have to be tracked or observed by the chaser in order to estimate its relative pose. Our developed theoretical study provides a useful tool to conduct simple experiments on pose estimation and RVD operations.

For future works, we suggest to include the study of GEO orbits for far maneuvers and the development of the navigation filter that includes an observer to estimate and predict the structure of motion. Consistently with this work, some practical experiments are being proposed at Center of Applied Space Technology and Microgravity (ZARM). The presented study shall be applied in the ZARM test bed facilities, taking into account the satellite dynamics simulator vehicle project entitled LUVEX [28]. That simulator includes the capability to perform, test and validate simple RVD operations using two vehicles. One of the vehicles shall carry a camera, so as to provide an environment to test the estimation pose algorithm, when estimating position and attitude of one of the vehicle. Both vehicles allow us to study formation flying in the Rbar and the V-bar plane.

\section{Acknowledgments}

This work was funded by the Coordination for the Professional Development of University Educated Personal CAPES, process BEX: $1326 / 05-8$, and the Center of Applied Space Technology and Microgravity - ZARM.

\section{References}

[1] D.G. Muff, Orbit determination requirements for synthetic aperture radar, in: Geoscience and Remote Sensing Symposium Proceedings, 1998 (IGARSS apos;98. 1998 IEEE International 2(6-10) (1998) 697-700).

[2] T.E. Rumford, Demonstration of autonomous rendezvous technology DART project summary, Proceedings of the SPIE 5088 (10) (2003) 10-19 doi:101117/12.498811.

[3] W.-K. Yoon, T. Goshozono, H. Kawabe, M. Kinami, Y.T.M. Uchiyama, M. Oda, T. Doi, Model-based space robot teleoperation of TS-VII, IEEE Transactions on Robotics and Automation 20 (3) (2004) 602-612.

[4] B. Albert Bosse, W. James Barnds, A. Michael Brown, N. Glenn Creamer, SUMO: Spacecraft for the Universal Modification of Orbits, Naval Research Laboratory, 2006.

[5] J.C. Diaz, M. Abderrahim, Visual Inspection System for Autonomous Robotic On-Orbit Satellite Servicing, ASTRA2006: 9th ESA Workshop on Advanced Space Technologies for Robotics and 
Automation, Netherlands, ESA Publications Division (European Space Agency), 2006.

[6] A. Cropp, P. Palmer, C.I. Underwood, Pose estimation of target satellite for proximity operations, in: Fourteenth Annual/USU Conference on Small Satellites, Utah, 2000.

[7] A. Cropp, P. Palmer, C.I. Underwood, Pose estimation and relative orbit determination of a nearby target microsatellite using passive imagery, in: Cranfield DCSSS Conference, 2002.

[8] F.Z. Qureshi, D. Terzopoulos, P. Jasiobedzki, A cognitive vision system for space robotics, in: Proceedings of the IAPR Conference on Machine Vision Applications, Japan, 2005.

[9] F.Z. Qureshi, D. Terzopoulos, P. Jasiobedzki, A cognitive vision for autonomous satellite rendezvous and docking, applications of computer vision workshop, in: European Conference of Computer Vision, Czech Republic, 2004.

[10] Ondrej Chum, The correspondence problem in perspective images, Ph.D. Thesis, CTU-CMP-2003-03, ISSN 1213-2365, Czech Technical University, Center of Machine Perception, 1993.

[11] E.M. Rocco, Tool for analysis and simulation of spacecraft trajectories in Keplerian orbit, Technical Report Nr: INT-DYN-TUZAR-001, Center of Applied Space Technology and Microgravity (ZARM), Bremen, Germany, 2007.

[12] R.R. Bate, D.D. Mueller, E.J. White, Fundamentals of Astrodynamics, 1st ed., Dover Publications, INC, New York, 1971 pp. 227-275, ISBN: 0-486-60061-0.

[13] E.M. Rocco, Tool for analysis and simulation of spacecraft trajectories in Keplerian orbit, Technical Report Nr: INT-DYN-TUZAR-002, Center of Applied Space Technology and Microgravity (ZARM), Bremen, Germany, 2007.

[14] Wigbert Fehse, Automated Rendezvous and Docking of Spacecraft, Cambridge Aerospace Series, 1st Edition, 2003, ISBN: 0521824923.

[15] Y. Ma, S. Soatto, J. Kosecka, S.S. Sastry, An Invitation to 3D Vision: From Images to Geometric Models, 1st Ed., Springer, ISBN 9780387008936, 2004.

[16] J. Heikkila, O. Silven, A four-step camera calibration procedure with implicit image correction, in: Computer Vision and Pattern Recognition, IEEE Computer Society Conference on Computer Vision, Puerto Rico, 1997, pp. 1106-1112, ISBN: 0-8186-7822-4.
[17] O.D. Faugeras, G. Toscani, Camera calibration for 3D computer vision, in: Proceedings of the International Workshop on Industrial Applications of Machine Vision and Machine Intelligence, Silken, Japan, 1987, pp. 240-247.

[18] L. Kavan, S. Collins, C. O’Sullivan, J. Zara, Skinning with Dual Quaternions, Symposium on Interactive 3D Graphics and Games, 2007.

[19] L. Kavan, S. Collins, C. O'Sullivan, J. Zara, Dual quaternions for rigid transformation blending, Technical Report, Trinity College, Dublin, 2006.

[20] K. Danilidis, Hand eye calibration using dual quaternions, International Journal of Robotics Research 18 (1999) 286-298.

[21] J.E. Dennis Jr., in: D. Jacobs (Ed.), Non-Linear Least Squares: State of the Art in Numerical Analysis, Academic Press, 1977, pp. 269-312.

[22] K. Levemberg, A method for the solution of certain problems in least-squares, Quarterly of Applied Mathematics 2 (1944) 164-168.

[23] D. Marquardt, An algorithm for least squares estimation of nonlinear parameters, SIAM Journal on Applied Mathematics (1963) 431-441.

[24] L.J. Cohon, Multiobjective Programming and Planning, Academic Press, New York, 1978333 pp.

[25] E.M. Rocco, M.L.O. Souza, A.F.B.A. Prado, Multi-objective optimization applied to satellite constellation I: formulation of the smallest loss criterion, in: IAC 2003, 54th International Astronautical Congress, Bremen, Germany, 2003.

[26] E.M. Rocco, M.L.O. Souza, A.F.B.A. Prado, Further application of the smallest loss criterion in the multi-objective optimization of a satellite constellations, in: IAC 2005, 56th International Astronautical Congress, Fukuoka, Japan, 2005.

[27] E.M. Rocco, M.L.O. Souza, A.F.B.A. Prado, Multi-objective optimization applied to satellite constellation II: initial applications of the smallest loss criterion, in: Fourth IWSCFF International Workshop on Satellite Constellations and Formation Flying, February 14-16, Sao Jose dos Campos, Brazil, 2005.

[28] Giuseppe Apriliano, Design of the testbed facility for the air cushion vehicle LUVEX, Technical Report, Center of Applied Space Technology and Microgravity (ZARM), 1999. 